\title{
Initiation à l'analyse et à la communication scientifique par la mise en place de projets tuteurés en L3 Physique à l'Université Montpellier II.
}

\author{
D.Laux ${ }^{1 *}$, P.Barate ${ }^{2}$, T.Guillet ${ }^{2}$, S.Juillaguet ${ }^{2}$, L.Konczewicz ${ }^{2}$, T.Taliercio ${ }^{1}$ \\ *Auteur correspondant : Didier Laux. \\ Didier.Laux@univ-montp2.fr \\ Département d'Enseignement de Physique (DEPHY). Bât 13. $1^{\text {er }}$ Etage. CC070. \\ Université Montpellier II. Place Eugène Bataillon. 34095 Montpellier. France. \\ ${ }^{1}$ : IES. Institut d'Electronique du Sud. UMR CNRS 5214. CC082. Université Montpellier II. \\ 2 : GES. Groupe d'Etude des Semiconducteurs. UMR CNRS 5650. CC074. Université Montpellier II.
}

\begin{abstract}
RESUME : Dans le cadre de la réforme du LMD (Licence - Master - Doctorat), et pour initier les étudiants à l'analyse scientifique, nous avons mis en place un enseignement sous la forme de projets tuteurés en L3 Physique à l'Université de Montpellier dans le Département d'Enseignement de Physique (DEPHY). La mise en place de ce module, les thèmes abordés, les modalités de contrôle des connaissances, le retour d'expérience sur 2 ans de fonctionnement, l'avis des étudiants sur cette unité d'enseignement sont présentés dans cette communication. Pour illustrer notre propos, nous mettons l'accent sur quelques thèmes qui ont motivé les étudiants et ont conduit à la réalisation de très bons projets.
\end{abstract}

Mots clés : projets tuteurés, L3 Physique, expérimentation, simulation, communication, analyse scientifique.

\section{INTRODUCTION}

L'objectif de ces projets tuteurés est de développer les capacités d'analyse scientifique et de communication par un travail de recherche encadré pour des étudiants du L3 Physique à Montpellier répartis en trois parcours: Physique fondamentale, Physique et Applications, Sciences Physiques et Chimie.

Les deux questions majeures qui se sont rapidement posées pour le montage de cette unité d'enseignement (UE) sont les suivantes :

- trouver suffisamment de sujets adaptés aux divers parcours et réalisables avec les moyens humains et matériels disponibles.

- trouver un mode de fonctionnement qui permette aux étudiants d'être à la fois assez autonomes et suffisamment encadrés par les enseignants.

Pour les sujets nous avons essayé de nous éloigner des sujets académiques classiques qui sont traités dans la plupart des TP pour tendre peu à peu vers des sujets qui sont abordés dans les laboratoires de recherche. Cela permet aux étudiants de prendre connaissance de l'activité scientifique des laboratoires. Etant donné que les étudiants ne disposaient pas toujours du niveau théorique requis pour traiter ces thèmes, leur projet a été divisé en deux parties : une partie formation (introduction aux notions de base, prise en main du matériel...) et une partie réalisation (autonomie des étudiants face à un problème donné).

\section{ORGANISATION GENERALE DE L'UNITE D'ENSEIGNEMENT}

Les étudiants sont répartis en binômes ou trinômes, chaque enseignant étant responsable de 5 trinômes en moyenne. Compte tenu de nos effectifs on constitue environ 40 bi-trinômes, répartis en 8 groupes. L'équipe pédagogique étant actuellement constituée de 6 enseignants, chacun est donc responsable d'un ou deux groupes (cf Fig 1.).

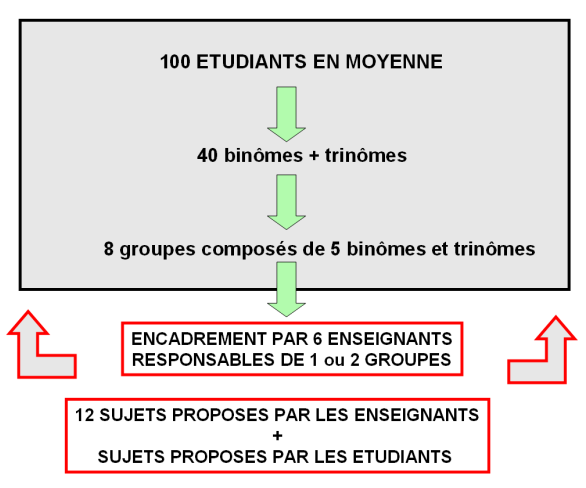

Fig 1. Organisation générale de l'UE 
Après présentation des sujets aux étudiants et attribution d'un sujet à chaque bi-trinôme (en fait, les étudiants classent les sujets par ordre de préférence et nous affectons les sujets en essayant de satisfaire le plus de monde possible), un cours magistral de 2 heures est effectué. Lors de ce cours, les notions suivantes sont abordées: comment faire une recherche bibliographique performante et pertinente, comment utiliser les ressources internet (sciencedirect, sudoc...), quels sont les pièges à éviter, comment présenter ses résultats (incertitudes sur les mesures), comment rédiger un rapport, comment présenter un travail à l'oral... L'accent a été mis sur les présentations utilisant des moyens informatiques : attention à ne pas faire « que du beau à l'oral » et privilégier le contenu scientifique.

A la suite de ce cours magistral une première séance pratique de 5 h est proposée aux étudiants. Cette séance nommée «manipulation 1 » est en réalité organisée sous forme de TP sur le sujet à traiter. Cette étape plutôt académique permet aux étudiants d'acquérir les notions de base sur le sujet et de prendre en main le matériel. Aucun compte rendu n'est restitué aux enseignants sur cette première séance. Une fois cette étape réalisée, les étudiants se concentrent sur la «manipulation 2 ». Elle dure également $5 \mathrm{~h}$. Les étudiants traitent une question plus générale sur le sujet en s'appuyant sur les acquis de la manipulation 1 . Cette question peut être soit proposée par les étudiants eux-mêmes soit suggérée par les enseignants si les étudiants sont « en manque d'inspiration ».

Entre les deux séances, nous invitons les étudiants à venir discuter avec les enseignants de l'équipe pédagogique pour bien fixer les objectifs de la manipulation 2, mettre à disposition du matériel spécifique au besoin, éclaircir certains points de blocage, qu'ils soient théoriques ou pratiques...

A l'issue de ces deux séances pratiques les étudiants rédigent leur rapport. Nous laissons à leur disposition la salle d'expériences pendant 2 jours supplémentaires pour que les groupes qui le souhaitent puissent compléter ou vérifier leurs mesures.

Ensuite vient la restitution des rapports écrits et enfin l'oral. Chaque bi-trinome présente son travail à l'oral pendant 20 minutes suivi de 10 minutes de questions. Le jury est composé de deux enseignants (enseignant responsable du groupe et un autre membre de l'équipe pédagogique). L'assistance est composée des membres du jury et de tous les étudiants du groupe de travail. La note finale est constituée à part égale de l'évaluation du rapport et de l'évaluation de l'oral.

Avec cette organisation les étudiants passent en moyenne 20 à 25 heures avec les enseignants. Le reste du temps est consacré à la recherche bibliographique, à la rédaction du rapport, à la préparation de l'oral...).
Nous estimons que ce travail représente en moyenne aussi 20 à 30h (cf Fig 2.)

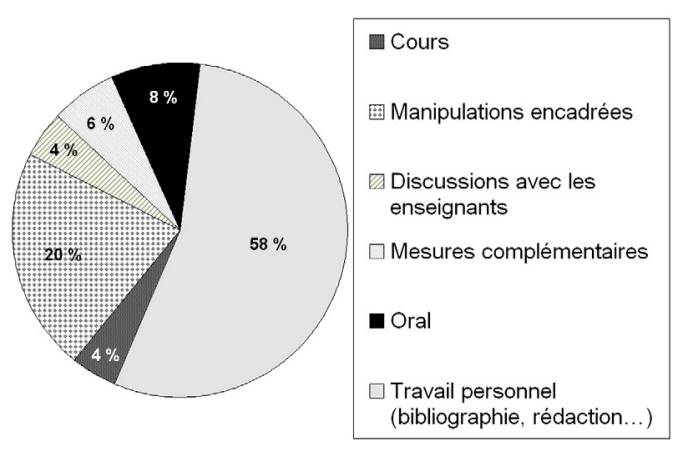

Fig 2. Découpage du travail effectué par les étudiants

\section{THEMES PROPOSES}

Compte tenu des trois parcours qui sont concernés, nous avons proposé à la fois des thèmes proches de la physique "pure», des thèmes plus proches de l'ingénierie, des thèmes à coloration EEA ainsi que des thèmes plus académiques qui correspondent peut-être mieux à des étudiants se dirigeant vers les concours d'enseignement comme le CAPES ou l'agrégation.

Dans tous les cas notre but est que les étudiants parviennent à acquérir des notions importantes mais aussi qu'ils parviennent à expliquer à leurs camarades et aux enseignants ces notions. Cet aspect pédagogique, trop souvent négligé au profit de la théorie constitue pour nous une priorité.

Nous proposons donc 12 thèmes au choix aux étudiants. Nous n'excluons pas des propositions de thèmes nouveaux par les étudiants à condition que nous disposions de matériel suffisant pour assurer le bon déroulement des expériences. Les 12 thèmes sont les suivants : tension superficielle et capillarité, optique des systèmes épais, diffraction des ondes ultrasonores, polarisation de la lumière, strioscopie et transformée de Fourier, CND et échographie, viscosité et viscosimètre, analyse thermique de composites par éléments finis, tuyaux sonores, filtrage et amplification en électronique, conversion analogique / numérique, conversion numérique / analogique.

\section{ZOOM SUR QUELQUES THEMES}

Parmi les divers thèmes proposés certains ont particulièrement motivé les étudiants qui, grâce à ces projets, ont abordé des notions dépassant largement le cadre de la licence. Aborder de telles notions sans l'aspect expérimental aurait été, à notre avis, très difficile. 


\subsection{Contrôle non destructif par ultrasons}

A l'issue de la première année de fonctionnement, le Département d'Enseignement de Physique nous a attribué une partie du budget pour acheter un générateur d'impulsions électriques. Ce type de générateur permet d'exciter des capteurs ultrasonores et donc de réaliser du contrôle non destructif par Ultrasons. Divers capteurs ont été prêtés par l'équipe MIRA (Micro-RhéoAcoustique) de l'Institut d'Electronique du Sud (UMR CNRS 5214) qui possède une expérience de plus de 20 ans dans le domaine des ultrasons. Ceci a permis d'intégrer aux projets tuteurés des thèmes actuellement traités en recherche et de faire découvrir aux étudiants notre travail de chercheur. Lors de la manipulation 1, les notions de base ont été enseignées aux étudiants : ondes longitudinales, transverses, modules élastiques... Le banc utilisé est présenté figure 3 .

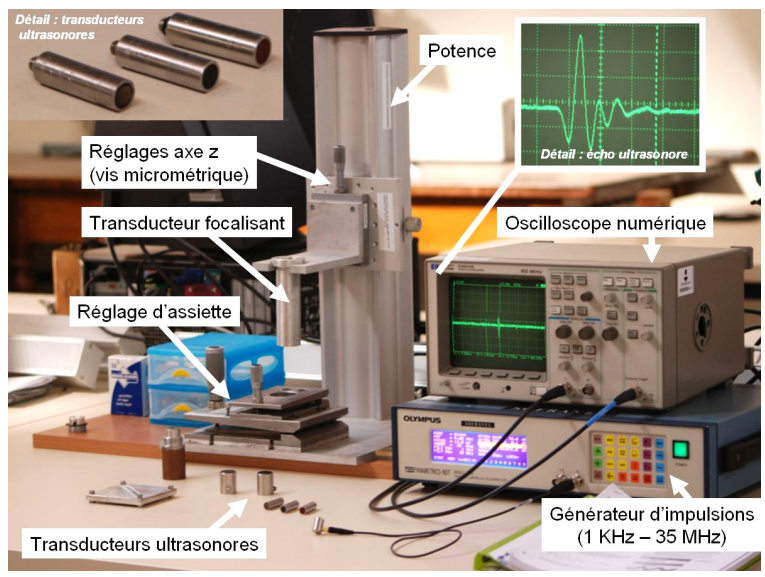

Fig 3. Banc ultrasonore monté pour les projets tuteurés

Pour la manipulation 2, l'accent a été mis sur la propagation des ondes transverses en milieu visqueux. Etant donné que le coefficient de réflexion sur une interface visqueuse est complexe, ceci induit un déphasage à la réflexion [1]. La visualisation et la quantification de ce phénomène assez «exotique » et contre intuitif a intéressé les étudiants. Par exemple ils ont pu mesurer ce très faible déphasage (environ $1 \mathrm{~ns}$ ) sur une interface verre / miel et le relier aux modules dynamiques $G^{\prime}$ et $G^{\prime}$ ' du miel. Ainsi à l'occasion de cette expérience les étudiants ont pu s'initier à la rhéoacoustique hautes fréquences, thème de recherche moderne dans divers laboratoires [2] [3]. Diverses discussions concernant la rhéologie nous ont conduit à évoquer des notions fondamentales dépassant largement le cadre du L3: équivalence temps température, relaxation, fluage...

\subsection{Conversion numérique / analogique (CNA) et analogique / numérique (CAN)}

Compte tenu de l'essor du codage numérique, les étudiants ont été particulièrement motivés par ces thèmes. Même si beaucoup d'entre eux n'avaient pratiquement pas de notion d'électronique dans leur cursus universitaire, les étudiants ont réussi d'une part à atteindre un niveau de compréhension du sujet poussé et d'autre part à explorer des sujets d'électronique annexes. Par ailleurs, comme nous proposons le CAN et le CNA, deux groupes particulièrement motivés ont décidé de collaborer pour partager leurs résultats : partant d'un signal analogique, un groupe a réalisé une conversion numérique. Ce signal numérique a ensuite été reconverti en signal analogique par un second groupe. Ceci leur a permis de mettre en évidence les notions de distorsion, d'erreur de conversion, de limitation du dispositif en fréquence et de comprendre l'importance d'ajuster précisément les performances des composants et de choisir le montage adapté à l'application envisagée.

\subsection{Viscosité et viscosimètre}

Pour ce thème, nous avons proposé aux étudiants de réaliser un viscosimètre à billes. Ce sujet très connu et assez académique ne présentait pas à priori de difficulté particulière. Pour la manipulation 1, le viscosimètre a été dimensionné, réalisé... Les premières mesures de vitesse de chute de la bille dans un liquide visqueux (du miel) ont conduit très rapidement à l'évaluation de la viscosité statique du miel à l'ambiante (environ 10 Pa.s). Pour la manipulation 2, les étudiants ont fait une étude en température. Après ajustement des courbes viscositétempérature par une loi de type Arrhenius ou WLF, l'énergie d'activation du miel a été évaluée. Il a été trouvé une valeur proche de $100 \mathrm{~kJ} \cdot \mathrm{mol}^{-1}$. Cette valeur est tout à fait conforme à ce que nous avons pu mesurer avec un rhéomètre. Cette valeur est par ailleurs conforme à celle donnée dans la littérature [4].

Pendant ce projet, diverses notions de mécanique des fluides ont été abordées: nombre de Reynolds, trainée... A ce propos les étudiants ont essayé de voir dans quelle mesure l'application de la relation de Stockes était justifiée. Ils ont alors quantifié les effets de bord, d'inertie grâce à la lecture d'un article et d'une thèse [5] [6]. Ce travail particulièrement intéressant a permis de corriger légèrement les premiers résultats obtenus. Ici encore nous soulignons le fait qu'à partir d'un sujet à priori très simple des étudiants très motivés on pu s'initier à des problématiques complexes non abordées généralement en L3. 


\section{EVALUATION DE L'UE}

A l'issu des oraux nous avons demandé aux étudiants leur avis sur l'UE de façon anonyme. Que ce soit l'an dernier ou cette année, les étudiants sont globalement satisfaits de l'UE.

Les points négatifs soulignés sont les suivants :

- manque de sujets,

- durée de l'UE trop courte pour approfondir vraiment

- affectation des sujets jugée trop arbitraire et aléatoire car faute de matériel certains étudiants se voient attribuer un sujet qu'ils ont classé en troisième position - bibliographie difficile à réaliser sur certains sujets.

Points positifs :

- UE différente des UE traditionnelles qui permet de découvrir la physique par soi-même

- découverte de sujets non traités en cours

- manipulations de longue durée

- discussions étudiants / enseignants enrichissantes

- une journée par semaine consacrée à ce travail dans l'emploi du temps

- couplage possible entre certains sujets : par exemple conversion Analogique / Numérique et Numérique / Analogique

\section{CONCLUSION ET PERSPECTIVES}

La mise en place de ces projets montre que les nouvelles façons d'enseigner plaisent globalement aux étudiants. Les notes qu'ils obtiennent sont correctes (moyennes de 12/20 environ à l'écrit ou à l'oral).

Le fait d'aborder des sujets de haut niveau, tout à fait nouveaux pour les étudiants, semble être un point très positif : ceci motive les étudiants qui perçoivent cela comme un challenge à relever. Côté enseignant l'expérience est aussi très enrichissante car on constate souvent que des trinômes peu motivés au début réalisent finalement de très bons projets.

Nous avons cependant constaté (surtout cette année) que les étudiants ont du mal à prendre du recul sur leur thème et restent souvent très " collés » au sujet. Il est aussi ressorti de discussions avec les étudiants que s'arrêter à l'oral était un peu dommage. Certains aimeraient faire des posters sur leur sujet, présenter leur travail à l'extérieur dans des congrès par exemple.

Une réflexion sur cet aspect valorisation des meilleurs projets est en cours de discussion au sein de l'équipe pédagogique. Dans ce contexte, nous pensons demander aux étudiants volontaires de participer aux journées portes ouvertes de l'Université. Par ailleurs inviter quelques étudiants à venir participer au prochain CETSIS pourrait être très intéressant. Les amener au CETSIS, afin de leur faire présenter euxmêmes leurs projets serait à notre avis une façon originale de valoriser leur travail. Ceci leur permettrait par ailleurs de rencontrer d'autres enseignants d'horizons divers.

De plus, les échanges enseignement - laboratoires ont été appréciés par les étudiants. Nous pensons renforcer ces collaborations l'an prochain: visite des laboratoires, couplage projet tuteurés - stages ... Nous espérons ainsi tirer le meilleur de l'apprentissage par projets à l'image d'expériences réalisées par certains collègues à plus grande échelle [7].

Pour finir, hormis les expériences sur le CND qui ont nécessité l'achat de matériel nouveau relativement onéreux, les autres projets peuvent être réalisés avec les moyens standards d'une salle de TP classique d'Université (PC, oscilloscopes, lasers, lentilles, composants électroniques, voltmètres...). Ceci présente l'avantage de pouvoir fonctionner avec un volant financier assez faible et montre aussi aux étudiants qu'il n'est pas forcément nécessaire d'avoir du matériel cher pour faire de la science. Ils prennent en particulier conscience que la qualité du matériel intervient surtout pour réduire les barres d'erreur.

\section{Bibliographie}

[1] P.Y. Longin, C. Verdier, M. Piau. "Dynamic Shear Rheology of high molecular weight Polydimethylsiloxanes : comparison of rheometry and ultrasound ", J.Non-Newtonian Fluid. Mech. 76 (1998) 213-232.

[2] A.Kulmyrzayev, D.J. McClements, "High frequency dynamic shear rheology of honey", J.Food.Eng. 45 (2000) 219.

[3] S.Dixon, B.Lanyon, " Phase change measurement of ultrasonic shear waves on reflection from a curing epoxy system". J.Phys.D : Applied Phys. 38 (2005) 4115.

[4] P.A.Sopade, P.Halley, B. Bhandari, B.D'Arcy, C.Doebler, N.Caffin. " Application of the Williams-Landel-Ferry model to the viscosity-temperature relationship of Australian honeys. " J.Food.Eng. 56 (2000) 67-75.

[5] M.Brizard, M.Megharfi, E.Mahé, C.Verdier "Un viscosimètre absolu pour la mesure de la viscosité des fluides". Revue Française de Métrologie. №10 Volume 2007-2.0

[6] Matthieu Brizard. " Développement et étude d'un viscosimètre absolu à bille". Thèse de doctorat. Université Joseph Fourier. Grenoble I. 20 Mai 2005.

[7] Damien Grenier, Saîd Belkouch, Khalid Faitah, Driss Yousfi, Bruno Dehez, Laurent de Vroey, Christophe Vloebergh, Eric Richard. " Apprentissage par projet autour d'un robot mobile: partage d'expériences entre l'Europe et le Maroc ". j3eA. Vol 7. Hors Série. Special CETSIS 2007. 